\title{
Adaptive Phase Measurements in Linear Optical Quantum Computation
}

\author{
T. C. Ralph $\uparrow \S$, A. P. Lund $\dagger$ and H. M. Wiseman $\ddagger$ \\ $\dagger$ Centre for Quantum Computer Technology, Department of Physics, The University \\ of Queensland, St Lucia QLD 4072 Australia \\ $\ddagger$ Centre for Quantum Computer Technology, School of Science, Griffith University, \\ Australia
}

\begin{abstract}
Photon counting induces an effective nonlinear optical phase shift on certain states derived by linear optics from single photons. Although this nonlinearity is nondeterministic, it is sufficient in principle to allow scalable linear optics quantum computation (LOQC). The most obvious way to encode a qubit optically is as a superposition of the vacuum and a single photon in one mode - so-called "singlerail" logic. Until now this approach was thought to be prohibitively expensive (in resources) compared to "dual-rail" logic where a qubit is stored by a photon across two modes. Here we attack this problem with real-time feedback control, which can realize a quantum-limited phase measurement on a single mode, as has been recently demonstrated experimentally. We show that with this added measurement resource, the resource requirements for single-rail LOQC are not substantially different from those of dual-rail LOQC. In particular, with adaptive phase measurements an arbitrary qubit state $\alpha|0\rangle+\beta|1\rangle$ can be prepared deterministically.
\end{abstract}

PACS numbers: 03.67.Lx, 03.65.Yz, 42.50.Lc, 02.30.Yy

$\S$ email:ralph@physics.uq.edu.au 


\section{Introduction}

Linear optical quantum computation (LOQC) employs linear networks to process encoded photonic qubits. The necessary non-linearities are induced via photon number resolving measurements which post-select desired outcomes. In principle, the results of these detections can be fed-forward in the network to produce a scaleable system. The first proposal of this type was made by Knill, Laflamme and Milburn (KLM) [1]. Since then a number of variations have been discussed [2, 3, 4, 5].

At the basic level the scheme is non-deterministic. A number of proof of principle experiments have now been performed at this level [6, 7, 8, 9]. Scale-up relies on implementing particular types of "teleported" gates with a specific failure mode (qubit measurement) which can be encoded against in various ways [1, 15, 10].

One point in common of all photonic LOQC proposals to date is that they exclusively employ photon counting for all measurements. It is of interest to investigate if other types of measurements can also play a role. Here we show that adaptive phase measurements 11] can be used to accomplish some useful tasks in LOQC schemes. Adaptive phase measurements are based on homodyne detection and thus the measurement outcome has a continuous spectrum — quite unlike the discrete values produced by a photon counter.

In this paper we will focus on single photon, "single-rail" schemes, where phasesensitive optical detection has the most obvious application. In these schemes the logical values of the optical qubits correspond to the occupation numbers of their respective field modes, i.e.: $|0\rangle_{L}=|0\rangle$ and $|1\rangle_{L}=|1\rangle$ where $|n\rangle$ is a single mode, $n$ photon Fock state. In contrast "dual-rail" schemes encode qubits between two modes as $|0\rangle_{L}=|01\rangle$ and $|1\rangle_{L}=|10\rangle$ where $|n m\rangle$ is the direct product of two single mode Fock states, with occupation numbers of $n$ and $m$ respectively. Basic non-deterministic gate operations have been described using single rail encoding [2] and experimental demonstrations of some basic operations have been made [12, 13, 14.

Before introducing adaptive measurements, it is useful to compare the two schemes, single-rail and dual-rail, when only photon counters are used (see Table 11). Dualrail requires single photons as resouces and photon counters for detection. Single-rail requires the same plus a supply of coherent states. Preparing arbitrary initial states and performing single qubit gates in dual-rail can be achieved with unit probability. However, in single-rail these operations are non-deterministic with low probability of success and require complicated interferometers. The two-qubit control sign gate is non-deterministic and of equal difficulty in both schemes. Because of the difficulty in performing single qubit operations this comparison suggests that single-rail schemes are much more difficult to implement than dual-rail schemes. We will revisit this comparison in the conclusion after adding adaptive phase measurements to our single-rail tool-kit. Before leaving the comparison it is worth noting the distinct effect of photon loss in each scheme. Photon loss in the dual-rail scheme moves the qubit outside the logical basis whilst in the single-rail scheme photon loss can be treated as a logical error. This means 
that standard error correction schemes could be used in a single-rail scheme to combat loss and other logical errors simultaneously. In dual-rail schemes a separate protocol targeted specifically at loss errors would be required. That said we note that an efficient coding against loss in dual rail has been described [15].

\begin{tabular}{||r||c|c||}
\hline \multicolumn{1}{||}{ Qubit } & Dual-Rail & Single-Rail \\
\hline State resources & $c_{0}|01\rangle+c_{1}|10\rangle$ & $c_{0}|0\rangle+c_{1}|1\rangle$ \\
\hline Detector resources & photon counters & photon counters \\
\hline Preparing a qubit & deterministic, free & prob., expensive \\
\hline Single qubit gates & deterministic, free & prob., expensive \\
\hline Two-qubit gates & prob., cheap & prob., cheap \\
\hline Photon loss & Outside encoding & Logical error \\
\hline$\Longrightarrow$ Q. Encoding & beyond standard & standard \\
\hline
\end{tabular}

Table 1. Comparison of the dual-rail and single-rail encodings prior to this work. Here 'prob.' stands for probabilistic and means can be done with probability less than unity. 'Free' means can be achieved with only one use of the resource to achieve a unit fidelity, 'cheap' means can be achieved with a finite number of resources (on average) to achieve unit fidelity and 'expensive' means unit fidelity can only be achieved asymptotically, with arbitrarily many resources. $|0\rangle$ and $|1\rangle$ are Fock states and $|\alpha\rangle$ represents a coherent state. Italics are used to indicate apparent disadvantages.

The paper is arranged in the following way. In the next section we will describe adaptive phase measurements and derive the relevant projector appropriate for single rail encoding measurements. In section 3 we will describe how a single rail superposition state can be deterministically produced from single photon number states using adaptive phase measurements. In section 4 we will consider another application; the reduction of dual-rail encoding to single rail encoding using adaptive phase measurements and hence describe how arbitrary rotations of single rail qubits can be implemented based on this technique. Finally in section 5 we will conclude.

\section{The Adaptive Phase Measurement}

In this section we show how the required phase measurements can be achieved using adaptive dyne detection. Here 'dyne' detection means detection using a large local oscillator in a coherent state, as in heterodyne and homodyne detection. This can be achieved with high efficiency [16] and we will assume an efficiency of unity. Consider a single mode (i.e. transformed-limited) pulse with an envelope $u(t) \geq 0$. This pulse shape $u(t)$ is assumed to be 0 for $t<0$ and $t>T$ and to be normalized so that $U(T)=1$, where

$$
U(t)=\int_{0}^{t} u(s) d s
$$


We assume a mode-matched local oscillator, that is, with the same pulse shape, but we note that in principle a CW local oscillator could be used, and the dyne current corrected electronically by multiplying it by the factor $\sqrt{u(t)}$. Let the phase of the local oscillator be $\Phi(t)$, which is fixed for homodyne detection and rapidly varying (linearly in time) for heterodyne detection. In the large local oscillator limit, the dyne current for times $0<t<T$ is given by

$$
I(t)=e^{-i \Phi(t)} b(t)+\text { H.c.. }
$$

Here $b(t)$ is proportional to the annihilation operator for the field incident upon the detector at time $t$, and is given by

$$
b(t)=u(t) a-u(t) \int_{0}^{t} \frac{\sqrt{u(s)} \nu(s)}{1-U(s)} d s+\sqrt{u(t)} \nu(t)
$$

Here $a$ is the annihilation operator for the pulse, while $\nu(t)$ is the annihilation operator for the localized vacuum incident upon the detector at time $t$. It obeys

$$
\left[\nu(t), \nu(s)^{\dagger}\right]=\delta(t-s)
$$

Note that in the mean we have simply $\langle b(t)\rangle=u(t)\langle a\rangle$. The equation (3) can be derived using the results of Ref. [17, generalizing the mode shape $\gamma e^{-\gamma t}$ of a freely decaying cavity to an arbitrary mode shape $u(t)$ by defining a time-dependent cavity decay rate $\gamma(t)=-(d / d t) \ln [1-U(t)]$.

Before considering adaptive dyne measurements, it is instructive to consider conventional dyne detection, such as homodyne detection. In this case $\Phi$ is a constant, and for a single-mode pulse we are interested only in the integrated current

$$
X=\int_{0}^{T} I(t) d t
$$

Using integration by parts [without relying on any properties of $\nu(t)$ ], this can be evaulated from Eq. (2) to be

$$
X=a e^{-i \Phi}+a^{\dagger} e^{i \Phi}
$$

the $\Phi$-quadrature of the pulse. What Eq. (6) means is that the probability density for the integrated current is

$$
\wp(X=x) d x=\langle x|\rho| x\rangle d x,
$$

where $|x\rangle$ is an eigenstate of $a e^{-i \Phi}+a^{\dagger} e^{i \Phi}$ normalized so that $\left\langle x \mid x^{\prime}\right\rangle=\delta\left(x-x^{\prime}\right)$ and $\rho$ is the system state.

In adaptive dyne detection, the phase $\Phi(t)$ is made to depend upon $I(s)$ for $s<t$. This was first treated quantitatively in Ref. [1]. It requires a real-time feedback loop with a time delay much smaller than the characteristic width of the pulse, as realized for the first time in Ref. [18. This leads to a nonlinear, non-Markovian equation for $I(t)$, and the problem is intractable in general. However, for the particular case of the following adaptive algorithm

$$
\Phi(t)=\int_{0}^{t} \frac{I(s) d s}{\sqrt{U(s)}},
$$


introduced in Ref. 11] and implemented in Ref. [18, the problem can be solved semianalytically [19] using the theory of generalized quantum measurement. Moreover, for the particular case where the state contains at most one photon then there is an analytical solution [11] involving a single integral of the current, just as in the example of homodyne detection above. It turns out that the integral is the the same as that used in the feedback (8):

$$
\Theta=\int_{0}^{T} \frac{I(t) d t}{\sqrt{U(t)}}-\frac{\pi}{2} .
$$

Unlike $X, \Theta$ cannot be represented by an operator in the system Hilbert space. But the equation analogous to Eq. (17) is simply

$$
\wp(\Theta=\theta) d \theta=\langle\theta|\rho| \theta\rangle d \theta / 2 \pi,
$$

where

$$
|\theta\rangle=|0\rangle+e^{i \theta}|1\rangle
$$

This adaptive dyne detection is useful for estimating the unknown phase of an optical pulse. But at the single-photon level we are more interested in its ability in state preparation. Say the system is entangled with other modes, so that the total state is $|\Psi\rangle$. Then the conditioned state of the other modes after the measurement yielding result $\theta$ is

$$
\langle\theta \mid \Psi\rangle\rangle / \sqrt{2 \pi}
$$

where the squared norm of this state is equal to the probability density for obtaining this outcome. We discuss such applications in the next section.

\section{Superposition State Preparation}

A basic qubit operation is the production of superposition states. For dual-rail systems arbitrary superposition states can be prepared using only linear optics, provided single photon states of a single mode can be prepared. Arbitrary single-rail superposition states, $\alpha|0\rangle+e^{-i \phi} \sqrt{1-\alpha^{2}}|1\rangle$, with $\alpha$ and $\phi$ real numbers, are not so easy to produce. Previous suggestions for deterministic production of such states involved non-linearities significantly larger than currently feasible. Alternatively, non-deterministic techniques based on photon counting [20, 2] have been described and experimentally demonstrated 13. but these have low probabilities of success. A non-deterministic scheme based on homodyne detection has also been demonstrated 14. We now show that it is possible to deterministically produce an arbitrary single-rail state from a single photon state using linear optics and adaptive phase measurements.

Consider first producing an equal superposition state. We begin by splitting a

single photon equally into two modes, so that $|\Psi\rangle\rangle=(|0,1\rangle+|1,0\rangle) / \sqrt{2}$. If we then do an adaptive phase measurement on the first mode we obtain a result $\theta$, which, from Eq. (10), is drawn randomly from $[0,2 \pi)$. From Eq. (12), the second photon is prepared 
in state $|-\theta\rangle$. Then by feedforward onto an phase modulator, this random phase can be removed, yielding deterministically the state

$$
(|0\rangle+|1\rangle) / \sqrt{2}
$$

This can be contrasted with the technique used in Ref. [14 that relied upon homodyne detection. There the second mode is prepared in a state with known phase, but with a random amplitude:

$$
(x|0\rangle+|1\rangle) / \sqrt{1+x^{2}}
$$

Here from Eq. (17) the probability distribution for $x$ is

$$
\wp(x) d x=e^{-x^{2} / 2} \frac{1+x^{2}}{2 \sqrt{2 \pi}} d x .
$$

The probability for obtaining $x=1$ is infinitesimal. A finite probability of success necessarily leads to a drop in fidelity.

Our procedure is easily generalized to the production of an arbitrary superposition state. The single photon state is now mixed with a vacuum state on a beamsplitter with intensity reflectivity $\eta$. The resulting evolution is

$$
|1\rangle|0\rangle \rightarrow \sqrt{\eta}|1\rangle|0\rangle+\sqrt{1-\eta}|0\rangle|1\rangle
$$

We then make an adaptive phase meansurement on the reflected output port and introduce a phase delay $\psi$ to the transmitted mode. If the result of the adaptive phase measurement is $\theta$, the conditional state of the transmitted mode is, from Eq. (12),

$$
\sqrt{\eta}|0\rangle+e^{-i(\theta+\psi)} \sqrt{1-\eta}|1\rangle .
$$

By choosing $\sqrt{\eta}=\alpha$ and $\psi=\phi-\theta$ an arbitrary state can be produced deterministically.

\section{Quantum Gates using Adaptive Phase Measurements}

We have shown that arbitrary single rail, single qubit states can be produced deterministically from single photon states using adaptive phase measurements. For quantum processing however we require the ability to impose arbitrary rotations on unknown input states. This is a more difficult problem as it requires a Hadamard gate defined by the transformations $|0\rangle \rightarrow(|0\rangle+|1\rangle) / \sqrt{2}$ and $|1\rangle \rightarrow(|0\rangle-|1\rangle) / \sqrt{2}$. A Hadamard transformation plus the phase rotations that come from propagation allow arbitrary rotations. A non-deterministic Hadamard transformation for single rail qubits based on photon counting has previously been described 22. However, the success probability of this construction was very low. Here we show that a Hadamard transformation based on a combination of photon counting and adaptive phase measurements, whilst still non-deterministic, can be much more efficient.

The key observation underlying this new construction is that a deterministic mapping of dual rail encoding into single rail encoding can be achieved using adaptive phase measurements. Consider the arbitrary dual rail qubit $\alpha|01\rangle+e^{-i \phi} \sqrt{1-\alpha^{2}}|10\rangle$. Suppose an adaptive phase measurement is made on the second rail of the qubit giving 


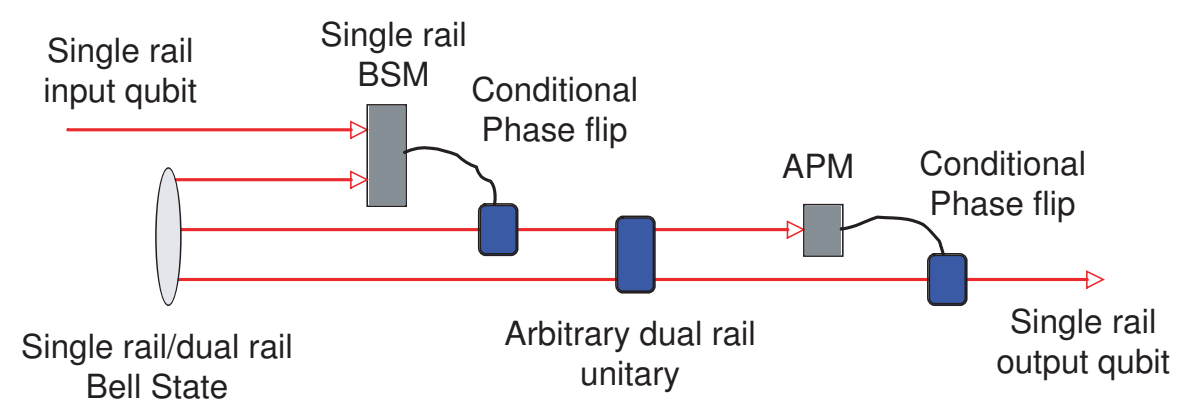

Figure 1. A schematic representation of the application of an arbitrary single qubit unitary to a single rail qubit. The single rail qubit is teleported onto a dual rail qubit, the unitary is applied, then an adaptive phase measurement is used to convert back to a single rail qubit. BSM $\equiv$ Bell state measurement. APM $\equiv$ Adaptive phase measurement. All operations are deterministic except the Bell state measurement which succeeds $50 \%$ of the time.

the result $\theta$ and a phase delay of $-\theta$ is subsequently imposed on the remaining rail of the qubit. The resulting state of the remaining rail is $\alpha|0\rangle+e^{-i \phi} \sqrt{1-\alpha^{2}}|1\rangle$, which is a single rail qubit with the same logical value as the original dual rail qubit.

What about the reverse operation? Is it possible to go from a single rail encoded qubit to a dual rail encoding? It does not appear to be possible to do this deterministically with only linear optics. However a non-deterministic transformation is possible using teleportation. The idea is to teleport between encodings. Dual rail teleportation can be achieved using the dual rail Bell state $|01\rangle|10\rangle+|10\rangle|01\rangle$. (We are ignoring normalization for convenience.) Single rail teleportation can be achieved using the single rail Bell state $|0\rangle|1\rangle+|1\rangle|0\rangle$. In both cases only two of the four Bell states can be identified with linear optics so the teleportation works $50 \%$ of the time. Now suppose we take a dual rail Bell state and use an adaptive phase measurement to project one of its arms into a single rail state. We obtain the state $|0\rangle|10\rangle+|1\rangle|01\rangle$ which is Bell entanglement between dual and single rail qubits. If we now perform a Bell measurement between the single rail half of the entanglement and an arbitrary single rail qubit then (when successful) the qubit will be teleported onto the dual rail part of the entanglement, thus converting a single rail qubit into a dual rail qubit.

We now have a way of (non-deterministically) performing an arbitrary rotation on an unknown single rail qubit. The idea is depicted schematically in Fig.1. First we teleport the single rail qubit onto a dual rail qubit. Then we perform an arbitrary rotation on the dual rail qubit. We then use an adaptive phase measurement to transform the dual rail qubit back to a single rail qubit. The only non-deterministic step is the Bell measurement in the teleportation thus the arbitrary rotation will succeed $50 \%$ of the time. This is a major improvement in success probability over previous schemes.

The fundamental 2 qubit operation, the control-sign gate, used in dual rail implementations is in fact a single rail gate [21]. The combination of arbitrary single qubit rotations and the control-sign gate form a universal set. 


\section{Conclusion}

We have shown that an adaptive phase measurement, when applied to an input state containing at most one photon, effectively measures the overlap of the input state with the measurement state $|0\rangle+e^{i \theta}|1\rangle$, where $\theta$ is the measurement outcome. We have applied this measurement technique to the deterministic preparation of arbitrary superposition states of single-rail photonic qubits. Previously it was thought that large non-linearities would be required for such operations. We have also applied the technique to the application of single qubit quantum gates for single rail qubits and described a more efficient (though still non-deterministic) protocol. The increase in efficiency when using adapative phase measurements with single-rail qubits is summarised in Table 2 (c.f. Table 1).

To summarize, this work introduces a new tool to the LOQC tool-box and increases the practicality of demonstrating and using single rail photonic qubits. Other applications for real-time feedback control techniques in LOQC remain to be explored.

\begin{tabular}{||r||c|c||}
\hline \multicolumn{1}{||r||}{ Qubit } & Dual-Rail & Single-Rail \\
\hline State resources & $c_{0}|01\rangle+c_{1}|10\rangle$ & $c_{0}|0\rangle+c_{1}|1\rangle$ \\
\hline Detector resources & $|1\rangle$ & $|1\rangle,|\alpha\rangle$ \\
\hline Preparing a qubit & PC & PC, $A P M$ \\
\hline Single qubit gates & deterministic, free & deterministic, free \\
\hline Two-qubit gates & prob., cheap & prob., cheap \\
\hline Photon loss & Outside encoding & Logical error \\
\hline$\Longrightarrow$ Q. Encoding & beyond standard & standard \\
\hline
\end{tabular}

Table 2. Comparison of the dual-rail and single-rail encodings considering this work. This table is the same format as Table 1 but photon counters has been abbreivated to PC. This time, italics indicate changes from Table 1 including adapative phase measurements (APM) eliminates the resource problems in preparing an arbitrary qubit and performing single qubits gates.

\section{Acknowledgments}

This project was supported by the Australian Research Council and the Queensland State Government.

\section{References}

[1] E. Knill, R. Laflamme, and G. J. Milburn, Nature, 404, 48 (2001).

[2] A. P. Lund, T. C. Ralph, Phys. Rev. A, 66, 032307 (2002).

[3] J. D. Franson, M. M. Donegan, M. J. Fitch, B. C. Jacobs, and T. B. Pittman, Phys. Rev. Lett. 89, 137901 (2002). 
[4] T. C. Ralph, A. Gilchrist, G. J. Milburn, W. J. Munro, and S. Glancy, Phys. Rev. A 68, 042319 (2003).

[5] M. A. Nielsen, Phys. Rev. Lett. 93, 040503 (2004).

[6] T. B. Pittman, M. J. Fitch, B. C. Jacobs, and J. D. Franson, Phys. Rev. A68, 032316 (2003).

[7] J. L. OBrien, G. J. Pryde, A. G. White, T. C. Ralph and D. Branning, Nature 426, 264 (2003).

[8] S. Gasparoni, J-W. Pan, P. Walther, T. Rudolph, and A. Zeilinger Phys. Rev. Lett. 93, 020504 (2004).

[9] Z. Zhao, A.-N. Zhang, Y.-A. Chen, H. Zhang, J.-F. Du, T. Yang, and J.-W. Pan Phys. Rev. Lett. 94, 030501 (2005).

[10] A. J. F. Hayes, A. Gilchrist, C. R. Myers, T. C. Ralph, J. Opt. B: Quantum Semiclass. Opt. 6, 533 (2004).

[11] H.M. Wiseman, Phys. Rev. Lett. 75, 4587 (1995).

[12] E. Lombardi, F. Sciarrino, S. Popescu, and F. De Martini Phys. Rev. Lett. 88, 070402 (2002).

[13] A. I. Lvovsky and J. Mlynek Phys. Rev. Lett. 88, 250401 (2002).

[14] S. A. Babichev, B. Brezger, and A. I. Lvovsky Phys. Rev. Lett. 92, 047903 (2004).

[15] T.C.Ralph, A.J.F.Hayes and A.Gilchrist, quant-ph/0501184 (2005).

[16] H. A. Bachor and T. C. Ralph, A Guide to Experiments in Quantum Optics 2nd Ed., Wiley-VCH, Wenheim (2004).

[17] H. M. Wiseman, Quantum Semiclass. Optics 7, 569 (1995).

[18] M.A. Armen et al., Phys. Rev. Lett. 89, 133602 (2002).

[19] H. M. Wiseman and R. B. Killip, Phys. Rev. A 57, 2169 (1998).

[20] D. T. Pegg, L. S. Phillips and S. M. Barnett, Phys. Rev. Lett.81, 11604 (1998).

[21] A. P. Lund, T. B. Bell, and T. C. Ralph, Phys. Rev. A 68, 022313 (2003). 